\title{
High-intensity focused ultrasound enhances the effect of bufalin by inducing apoptosis in pancreatic cancer cells
}

This article was published in the following Dove Medical Press journal:

OncoTargets and Therapy

\author{
Zhouyu Ning ${ }^{1,2, *}$ \\ Zhenfeng Zhu ${ }^{1,2, *}$ \\ Haiyong Wang ${ }^{1,3}$ \\ Chenyue Zhang ${ }^{1,2}$ \\ Litao $\mathrm{Xu}^{1,2}$ \\ Liping Zhuang ${ }^{1,2}$ \\ Xia Yan ${ }^{1,2}$ \\ Dan Wang ${ }^{1,2}$ \\ Peng Wang ${ }^{1,2}$ \\ Zhiqiang Meng ${ }^{1,2}$ \\ 'Department of Integrative Oncology, \\ Fudan University Shanghai Cancer \\ Center, Shanghai, China; ${ }^{2}$ Department \\ of Oncology, Shanghai Medical \\ College, Fudan University, Shanghai, \\ China; ${ }^{3}$ Department of Radiotherapy, \\ Shandong Cancer Hospital, \\ Shandong, China \\ *These authors contributed equally \\ to this work
}

Correspondence: Zhiqiang Meng Department of Integrative Oncology, Fudan University Shanghai Cancer Center, 270 DongAn Road, Shanghai 200032, China

Tel +862164175590

Fax +86 2I 64437657

Email mengzhq@yeah.net
Purpose: High-intensity focused ultrasound (HIFU) has the potential to be an effective therapeutic strategy for pancreatic cancer (PC). However, owing to the high malignancy and poor prognosis of $\mathrm{PC}$, the use of HIFU therapy alone is not sufficient to impair the progression of PC. Bufalin, a compound extracted from traditional medicine, is known to inhibit the growth and progression of PC cells. However, the effect of the combination therapy of HIFU plus bufalin (HIFU+bufalin) is still uncertain.

Materials and methods: A colony formation assay and flow cytometry were performed to measure the growth and induction of apoptosis in PC cells. Western blotting was used to explore the potential mechanism of HIFU and bufalin therapy. The in vivo efficacy of HIFU+bufalin was tested in a $\mathrm{MiPaCa}_{2}$ xenograft model.

Results: Bufalin inhibited the growth of PC cells more obviously compared to HIFU. Combining bufalin with HIFU further decreased the growth of $\mathrm{MiaPaCa}_{2}$ cells compared with single therapy in vitro. Flow cytometry results showed that the percentage of surviving $\mathrm{MiaPaCa}_{2}$ cells in the bufalin-treated group and the HIFU-treated group was approximately three-fold and two-fold higher than in the HIFU+bufalin-treated group. Contrasting results were found in Panc-1 cells. Biochemical analysis revealed that HIFU+bufalin treatment elevated PARP expression and increased caspase- 8 activation in $\mathrm{MiaPaCa}_{2}$ and Panc- 1 cells. HIFU+bufalin significantly reduced the growth of $\mathrm{MiaPaCa}_{2}$ tumors compared with HIFU or bufalin treatment alone. HIFU+bufalin treatment decreased Ki67 staining and increased activated caspase- 3 and caspase 8 staining, when compared with HIFU or bufalin treatment alone in mouse tumors.

Conclusion: HIFU enhanced the effect of bufailn by inducing apoptosis in PC cells. A combination of HIFU and bufalin may be employed as an alternative therapeutic strategy for PC.

Keywords: extracorporeal shockwave therapy, bufalin, apoptosis, pancreatic neoplasms

\section{Introduction}

Pancreatic cancer (PC) is one of the most common cancers, and was the fourth leading type of cancer responsible for new cancer deaths in the USA in 2017. 1,2 Surgical resection is the most effective methods to improve the prognosis for PC. However, most patients undergoing this procedure will have an advanced stage of cancer, owing to the insidious nature of the onset of the disease and its rapid progress.

More than $80 \%$ of the patients are unsuitable for surgery at the time of diagnosis, and the 5-year survival rates of PDAC (pancreatic ductal adenocarcinoma) is in the 
range of $8 \%-10 \% .^{3,4}$ Therefore, novel therapeutic strategies are urgently needed for advanced PC.

High-intensity focused ultrasound (HIFU) is a noninvasive technique that has been used for the treatment of both benign and malignant tumors. In HIFU, an ultrasound (US) beam propagates through soft tissue as a high-frequency pressure wave. The US beam is focused on a small target volume, resulting in the conversion of energy into heat at this site. This increase in temperature causes coagulative necrosis and protein denaturation within only a few seconds. ${ }^{5,6}$ HIFU is capable of providing a completely non-invasive treatment without causing damage to the direct adjacent tissues..$^{5}$ In addition, the safety and efficacy of HIFU in ablation therapy for benign and malignant tumors have been investigated in patients, and results have indicated that HIFU may be a safe and effective method for tumor treatment..$^{7-9}$ Our previous study revealed that the use of a multimodal treatment approach (the combined therapy of HIFU and chemotherapy, with or without radiotherapy) could improve survival of patients with advanced PC, and that repeated HIFU resulted in a survival benefit without increased risk. ${ }^{10}$ However, the mechanism of HIFU for the treatment of advanced PC is unclear.

Bufalin is the major digoxin-like component of Chan $\mathrm{Su}$, a traditional Chinese medicine obtained from the skin and parotid venom glands of a toad. Many studies have focused on the anticancer activities of bufalin, which have been proven to inhibit cell proliferation and angiogenesis, induce differentiation and apoptosis, reverse drug resistance, alter gene expression in tumor cells, and regulate the body's immune system. ${ }^{11-16}$ In our previous studies, it was indicated that bufalin significantly inhibited the proliferation, invasion, and metastasis of hepatocellular carcinoma cells through the PI3K/AKT/mTOR/HIF-1 $\alpha$ pathway. ${ }^{17}$ However, there is no evidence to show whether bufalin could enhance the sensitivity of HIFU.

In this study, we examined the therapeutic effects of HIFU together with bufalin (HIFU+bufalin) in PC cells and xenograft models. Our data revealed that HIFU+bufalin treatment further deduced the growth and increased the apoptosis of PC cells in vitro and in vivo, comparing to bufalin or HIFU treatments alone. Tumors treated with HIFU+bufalin showed significantly more staining cells against activated caspase 3 and caspase 8 antibodies, and less staining cells against $\mathrm{Ki}-67$ antibodies in comparison with HIFU or bufalin-treated tumors. Our results suggest the benefits of combination therapy of HIFU and bufalin for the treatment of PC.

\section{Materials and methods Cell culture and reagents}

$\mathrm{PC}$ cell line $\mathrm{MiaPaCa}_{2}$ and Panc-1 cells were purchased from the Shanghai Institute of Biochemistry and Cell Biology and cultured in Dulbecco's Modified Eagle's Medium (DMEM) (HyClone Laboratories, Logan, UT, USA) supplemented with 10\% fetal bovine serum (FBS) (Sigma-Aldrich Co., St Louis, MO, USA), $1 \mathrm{mM}$ sodium pyruvate, 100 units $/ \mathrm{mL}$ penicillin, and $100 \mu \mathrm{g} / \mathrm{mL}$ streptomycin (Hyclone). All cell lines were cultured in a humidified tissue incubator at $37^{\circ} \mathrm{C}$, with an atmosphere of $5 \% \mathrm{CO}_{2}$ and $95 \%$ air. Bufalin was purchased from Sigma-Aldrich Co. and dissolved in DMSO.

\section{HIFU device}

The US-guided HIFU device was the Model-JC HIFU system (Haifu Technology, Chongqing, China). This device is CE-marked for the application to solid tumors in soft tissues, including pancreatic, liver, kidney, breast, and bone tumors, uterine fibroids, and adenomyosis. This device uses a 3.5 MHz diagnostic transducer and a $1.6 \mathrm{MHz}$ therapeutic imaging transducer., 8

\section{HIFU therapy}

Cells $\left(2 \times 10^{6}\right.$ cells $)$ were placed in a $15 \mathrm{~mL}$ centrifuge tube. The probe was covered with a sterile membrane and then immersed approximately $1 \mathrm{~cm}$ below the liquid level for 60 seconds. Pulses were applied at ISAL $=589 \mathrm{~W} / \mathrm{cm}^{2}$, with a pulse duration of 500 milliseconds. All operations were performed in a biosafety cabinet.

Animals were anesthetized by the intravenous injection of pentobarbital sodium $(50 \mathrm{mg} / \mathrm{kg})$. After narcosis, the animals were maintained in the lateral decubitus position to allow the tumor to be viewed clearly using ultrasonography. Ultrasound coupling gel was applied to eliminate air pockets trapped between the probe and the skin of the nude mice. The hypoechoic tumor tissue was treated point-by-point: therapy depth was $6 \mathrm{~mm}$, and treatment was performed in a horizontal mode with one layer. In all cases, pulses were applied at $\mathrm{ISAL}=589 \mathrm{~W} / \mathrm{cm}^{2}$ with a pulse duration of 500 milliseconds; there was an exposure separation of 1 second between each treatment point. The interval distance between treatment points was $1 \mathrm{~mm}$. Real-time ultrasound was used to target the tumor by moving the integrated probe, and the tumor was divided into $5 \mathrm{~mm}$ thick slices using ultrasound images and ultrasound scanning with a continuous HIFU beam and sweeping from the deep to the shallow regions of the tumor; the targeted regions in each slice of the tumor were completely ablated. If a hypoechoic region was observed 
after treatment, treatment was repeated until the region was hyperechoic.

\section{Colony formation assay}

Cells in the logarithmic growth phase were plated at 2,000 cells/well in a 6-well plate. Before seeding, cells in the HIFU and HIFU+bufalin groups were treated with HIFU. After 12 hours, bufalin was added into the medium at a final concentration of $20 \mathrm{nM}$ in the bufalin and HIFU+bufalin groups. Fresh medium was replaced every 2 days. After 2 weeks, the cells were fixed and stained with $0.5 \%$ crystal violet for 20 minutes at room temperature. Excess crystal violet was removed by three washes with water, the plates were dried, and images were captured by using a microscope. The number of cell colonies was calculated by using ImageJ software. Each experiment was performed in triplicate.

\section{Analysis of cell apoptosis}

Cells in the logarithmic growth phase were plated at a density of $5 \times 10^{5}$ cells/well in a 6 -well plate. Before seeding, cells in the HIFU and HIFU+bufalin groups were treated with HIFU. After 12 hours, bufalin was added into the medium at a final concentration of $20 \mathrm{nM}$ in the bufalin and HIFU+bufalin groups. After 72 hours of different treatments, the cells were harvested, centrifuged, and washed twice with cold $0.1 \mathrm{M}$ PBS at 2,000 rpm for 5 minutes, then suspended in $100 \mu \mathrm{L} 1 \times$ binding buffer. Next, cells were incubated with $5 \mu \mathrm{L}$ Annexin $\mathrm{V}$ and $5 \mu \mathrm{L}$ 7-ADD for 15 minutes at $25^{\circ} \mathrm{C}$ in a dark room. Finally, each sample was injected with $400 \mu \mathrm{L}$ $1 \times$ binding buffer, and the phase distribution of the cell cycle was determined by using flow cytometry and an Annexin V-FITC apoptosis detection kit I (BD Biosciences, San Jose, CA). Then cell apoptosis was assessed by CellQuest software (BD, Franklin Lakes, NJ, USA). Each experiment was performed in triplicate.

\section{Animal studies}

All animals received humane care in accordance with the principles described in the "Guide for the Care and Use of Laboratory Animals" issued by the National Institutes of Health. All procedures performed in studies involving animals were approved by the Institutional Animal Care and Use Committee of Fudan University. BALB/C nude mice were purchased from Shanghai SLAC laboratory animal center and were housed in a pathogen-free environment under controlled conditions. Human pancreatic $\mathrm{MiaPaCa}_{2}$ cancer cells were cultured in Dulbecco's Modified Eagle's Medium (Thermo Fisher Scientific, Waltham, MA, USA) supplemented with $10 \%$ FBS and $1 \%$ penicillin/streptomycin. For the implantation of donor mice, $1 \times 10^{8}$ cells $/ \mathrm{mL}$ were suspended in culture medium, and $200 \mu \mathrm{L}$ of cell suspension was inoculated under the skin of the right flank in each mouse. When the tumor had grown to a volume of approximately $1,500 \mathrm{~mm}^{3}$, the tumor was harvested, cut into $2 \mathrm{~mm}^{3}$ pieces, and inserted subcutaneously into the right flank of each mouse; subsequently, the skin was closed with a wound clip. Wound clips were removed 10-14 days post-implantation, and the tumors were measured every 3 days. When the average measured tumor volume (calculated according to the formula: (length $\times$ width $\times$ height)/2) reached approximately 130 $\mathrm{mm}^{3}$, the mice were randomized into groups, and treatment was administered. The average starting tumor volume per group was similar in all groups. Tumor growth was monitored by caliper measurement every 3 days. On Day 14, the mice were randomized and treated with vehicle (Normal saline, i.p, qd), HIFU (Day 14), bufalin (1.5 mg/kg, i.p, qd), and HIFU+bufalin for 21 days. Then the mice were sacrificed, and tumors were detected and stored in $10 \%$ formalin for $\mathrm{HE}$ and IHC staining.

\section{Western blot analysis}

Cells were seeded at $2 \times 10^{5}$ cells/well in 6-well plates, allowed to adhere for 12 hours, and treated with bufalin for 24 hours. Before seeding, the cells were treated with HIFU in the HIFU and HIFU+bufalin groups. Whole-cell lysates were prepared by using RIPA buffer. Protein concentrations in clarified lysates were quantified by using BCA Protein Assay Kit (Beyotime Institute of Biotechnology, Haimen, China) in accordance with the manufacturer's protocol. Equal amounts of cell lysates were loaded in $10 \%$ SDS-PAGE, transferred to a PVDF membrane (EMD Millipore, Billerica, MA, USA), immunoblotted with the appropriate primary and HRPconjugated secondary antibodies (Cell Signaling Technology, Inc., Danvers, MA, USA), and developed using enhanced chemiluminescence (ECL) reagent (Millipore Inc.). ECL signals were captured and analyzed by using Image Lab 4.0 software (Bio-Rad Laboratories Inc., Hercules, CA, USA). Beta-actin (Abcam Cambridge, UK) was used as the loading control. Antibodies recognizing cleaved caspase-3, caspase-8, caspase-9, poly (ADP-ribose) polymerase, Bak, and Bax were purchased from Cell Signaling Technology, Inc.

\section{Immunohistochemistry staining}

Sections were dewaxed, rehydrated, and subjected to heat-induced epitope retrieval in $10 \mathrm{mM}$ citrate buffer, $\mathrm{pH}$ 6.0, by using a microwave (high power for 5 minutes). 
The slides were immersed in $3 \% \mathrm{H}_{2} \mathrm{O}_{2}$ for 8 minutes at room temperature, blocked with $10 \%$ normal goat serum (Solarbio Life Sciences, Beijing, China) diluted in PBS for 20 minutes at room temperature, and incubated with Ki-67 mouse mAb $(1: 1,200)$, cleaved caspase 3 rabbit mAb (1:400), and caspase 8 (1:400) (Cell Signaling Technology, Inc.) overnight at $4^{\circ} \mathrm{C}$. For detection of these antibodies, polyperoxidase-conjugated anti-mouse/rabbit IgG Fab (ZSGB-BIO) was used. The slides were then incubated with 3,3'-diaminobenzidine chromogen DAB detection Kit (ZSGB-BIO) and counterstained with diluted hematoxylin for 2 minutes before mounting. To quantify the staining of Ki-67, caspase-8, and cleaved caspase-3, images from five random representative $400 \times$ fields per mouse tumor were captured by using a microscope. The positive cells in each field were counted, and the average of positive staining cells per field was presented. The representative images were shown from three independent experiments.

\section{Statistical analyses}

Quantitative data from three independent experiments were presented as the mean \pm SEM. ANOVA followed by post-hoc pairwise comparison using an unpaired $t$-test was used to analyze differences among treatment groups. All statistical tests were two-sided, and $P<0.05$ was considered to indicate statistical significance. Statistical analyses were performed using SPSS 19.0.

\section{Results}

HIFU, together with bufalin, impairs the growth of PC cells and induces the apoptosis of PC cells

Based on our published retrospective study, HIFU can prolong the overall survival of patients with PC, and bufalin is an effective drug for the inhibition of the growth of PC cells. We, therefore, wondered if HIFU and bufalin would have synergistic or antagonistic interactions. To test our hypothesis, colony forming assays were performed. The results revealed that bufalin treatment alone resulted in significant inhibition of cell growth in both cell lines, which was prior to the inhibitory effect of HIFU on both cell lines. Furthermore, treatment with HIFU+bufalin led to a greater reduction in cell growth than observed following treatment with either single agent in $\mathrm{MiaPaCa}_{2}$ cells (Figure 1A and B). These data implied that bufalin can enhance the effect of HIFU in inhibiting the growth of PC cells.
A previous study showed that HIFU ablation promotes the apoptosis of hepatocellular carcinoma cells and the effect has a close association with the change of p53 and bcl-2 expression. ${ }^{18}$ Therefore, we sought to determine whether HIFU induced PC cells to undergo apoptosis. By using a flow cytometry assay, we explored the apoptosis of pancreatic cells treated with HIFU, bufalin, and HIFU+bufalin. The drug concentration of bufalin and therapeutic power were $20 \mathrm{nM}$ and $2 \mathrm{~W}$, respectively. Bufalin was added into the cell culture medium for 72 hours, and HIFU treatment was performed before seeding cells. The results showed that the percentage of $\mathrm{MiaPaCa}_{2}$ cells surviving in the bufalin-treated group and the HIFU-treated group was approximately threefold and two-fold higher than that in HIFU+bufalin-treated group (Figure $1 \mathrm{C}$ and $\mathrm{E}$ ). Contrasting results were observed in Panc-1 cells (Figure 1D and F).

\section{Bufalin enhances the effect of HIFU by inducing expression of apoptosis-related proteins}

We confirmed that HIFU+bufalin induced apoptosis in pancreatic cells. However, we did not know which proteins were changed by the bufalin and HIFU treatment. We, therefore, detected the expression of apoptosis-related proteins by Western blotting. Increased cleavage of poly(ADPribose) polymerase (PARP) and caspase 8 were detected in the HIFU+bufalin treated groups in $\mathrm{MiaPaCa}_{2}$ cells, in comparison with the bufalin-treated group (Figure 2A), while cleaved caspase-3 was higher in the groups treated with bufalin and/or HIFU compared with the HIFU+bufalintreated group (Figure 2A). Similarly, Panc-1 cells in the HIFU+bufalin treatment group showed a higher PARP expression, as well as caspase- 8 expression, than in the bufalin-treated group (Figure 2B). Expression of cleaved caspase-3 in the HIFU+bufalin-treated group showed slightly higher than in the bufalin-treated group in Panc-1 cells (Figure 2B). These data indicated that caspase- 8 and PARP have an important role in bufalin and HIFU-induced apoptosis.

\section{Bufalin enhances in vivo sensitivity of HIFU in PC xenograft models}

Owing to its efficacy in the reduction of the growth of PC cells in vitro, the effect of HIFU on tumor growth was examined in a $\mathrm{MiaPaCa}_{2}$ xenograft model. The cells were suspended in the culture medium at a concentration of $1 \times 10^{8}$ cells $/ \mathrm{mL}$, and $200 \mu \mathrm{L}$ of cell suspension was inoculated under the skin of the right flank in mice. When it 

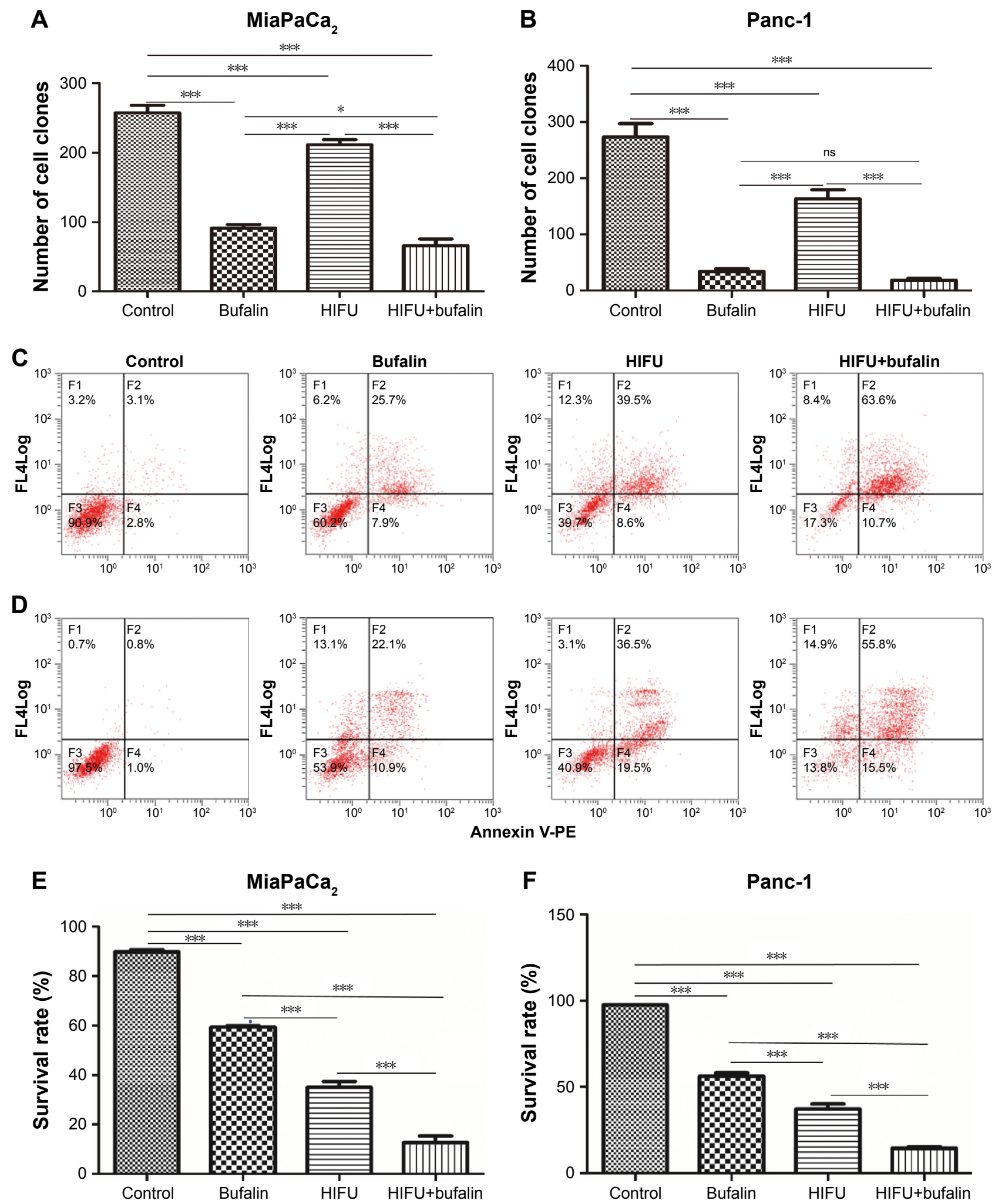

Figure I HIFU, together with bufalin, impairs the growth of PC cells and induces the apoptosis of PC cells.

Notes: The colony formation assay was used to assess the growth of pancreatic cells. HIFU, together with bufalin, inhibited the growth of $\mathrm{MiaPaCa}_{2}(\mathbf{A})$ and Panc-I (B) cells. Flow cytometric analysis was performed to evaluate the induction of apoptosis in PC cells. The proportion of $\mathrm{MiaPa}_{2}$ cells in apoptosis was highest in the HIFU+bufalintreated group than in the bufalin-treated or HIFU-treated groups (C). An increased number of apoptotic Panc-I cells was observed after HIFU+bufalin treatment than after treatment with bufalin or HIFU alone (D). The survival rates of cells in the control group, the bufalin group, the HIFU group, and the HIFU+bufalin group were $90.9 \%, 60.2 \%$, $39.7 \%$, and $17.3 \%(E)$, respectively. The survival rates of cells in the control group, the bufalin group, the HIFU group, and the HIFU+bufalin group were $97.5 \%, 53.9 \%, 40.9 \%$, and $13.8 \%$, respectively $(\mathbf{F})$. Error bars represent the mean \pm SEM of three independent experiments. Data shown are representative of three independent experiments. $* P<0.05$ and $* * * P<0.000$ I, one-way ANOVA.

Abbreviations: HIFU, high-intensity focused ultrasound; ns, nonsignificant. 

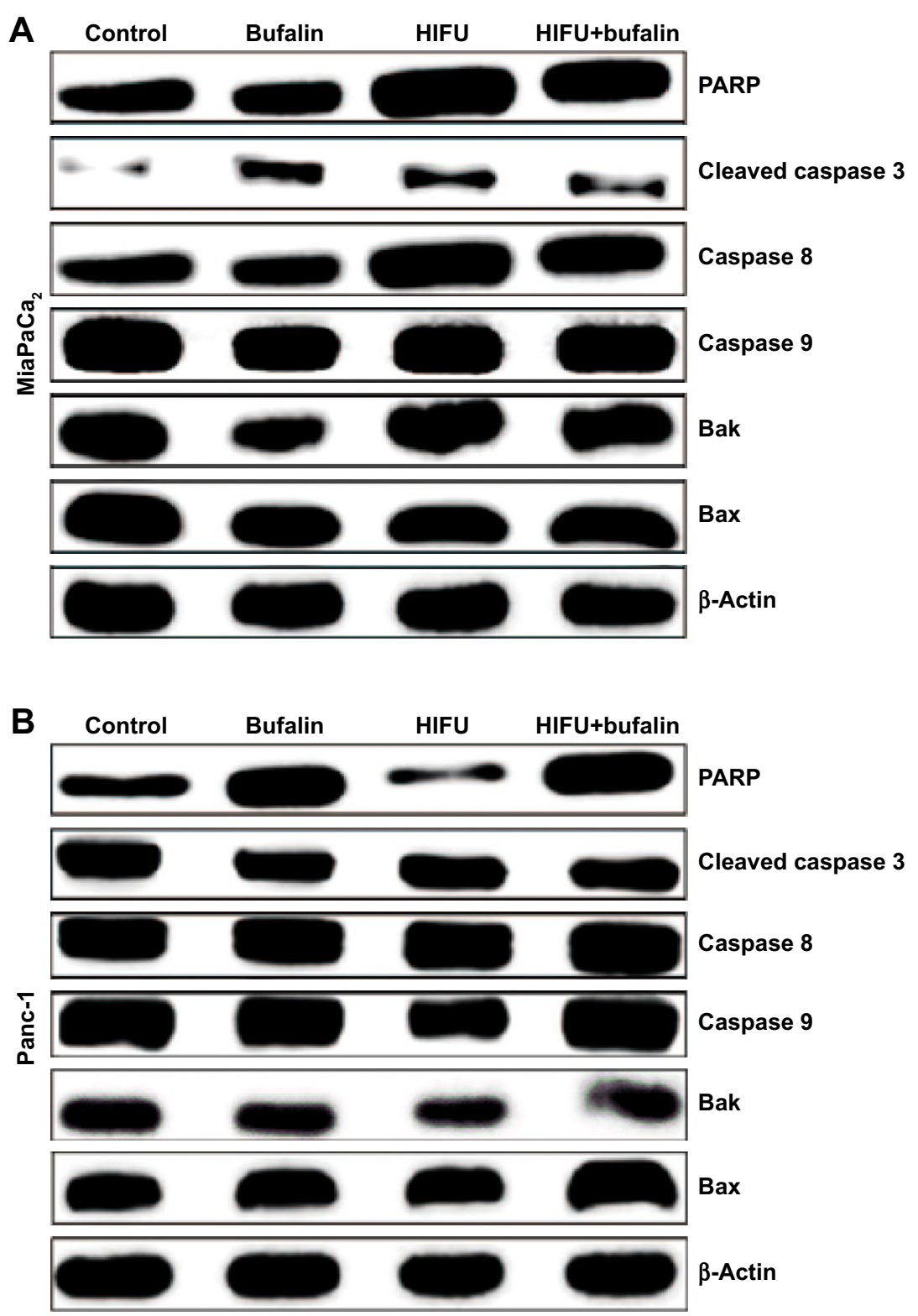

Figure 2 Bufalin, combining HIFU, induces expression of apoptosis-related proteins. PARP, cleaved caspase-3, caspase-8, caspase-9, Bax, and Bak protein expression in (A) $\mathrm{MiaPaCa}_{2}$ cells and (B) Panc-I cells were examined by Western blotting.

Note: Representative blots from three biological repeat experiments are shown.

Abbreviations: HIFU, high-intensity focused ultrasound; PARP, poly-ADP-ribose polymerase.

was grown in a donor mouse to a volume of approximately $1,500 \mathrm{~mm}^{3}$, the tumor was harvested, cut into $2 \mathrm{~mm}^{3}$ pieces, and inserted subcutaneously into the right flank of mice. The tumor growth was monitored by caliper measurement, and the mice were randomized into four groups for treatment with vehicle, bufalin, HIFU, or HIFU+bufalin at 2 weeks after cell injection. $\mathrm{MiaPaCa}_{2}$ tumors treated with vehicle control grew gradually, whereas bufalin treatment significantly reduced tumor growth. Similarly, HIFU treatment dramatically decreased $\mathrm{MiaPaCa}_{2}$ tumor growth. The addition of bufalin to HIFU caused a significant further reduction in the growth of $\mathrm{MiaPaCa}_{2}$ tumors compared with HIFU treatment alone. This result indicated that bufalin enhances the in vivo sensitivity of PC xenograft models to HIFU (Figure 3A and B).

\section{HIFU inhibits cell proliferation and increases cell apoptosis in $\mathrm{MiaPaCa}_{2}$ xenograft tumors}

Owing to the efficacy of HIFU in the induction of apoptosisrelated protein expression in vitro, immunohistochemical staining was performed to examine the expression of 

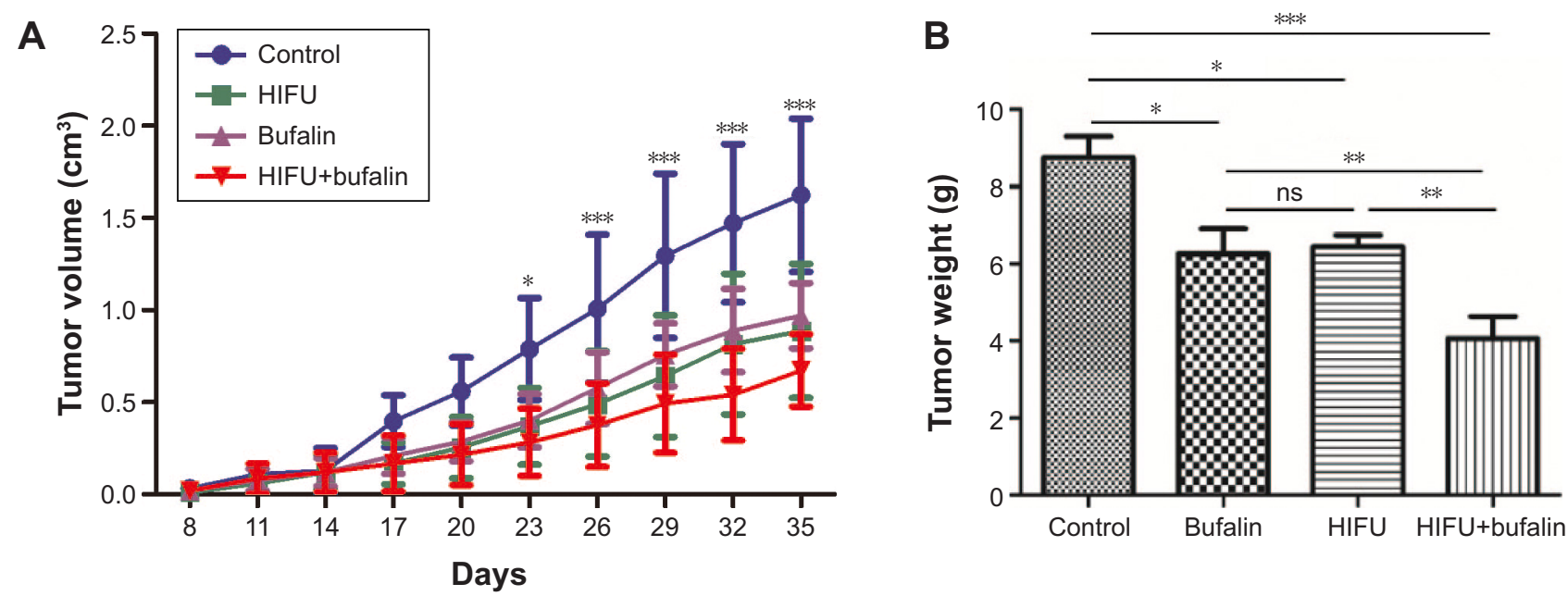

Figure 3 Bufalin enhances in vivo sensitivity of HIFU in PC xenograft models. MiaPaCa ${ }_{2}$ cells were injected into the right flank of BALB/C nude mice. After I4 days, the mice were randomized and treated with vehicle, HIFU, bufalin, and HIFU+bufalin for 21 days. The tumor growth in mice was monitored by caliper measurement every 3 days. (A) Tumor growth was shown as tumor volume. (B) Tumor weight in mice on Day 35 was compared in the four treatment groups. The data shown are representative of two independent experiments.

Notes: The error bars in (A) represent the SEM. $N=6$ mice for each group. $* P<0.05, * * P<0.0$ I and $* * * P<0.000$ I.

Abbreviations: HIFU, high-intensity focused ultrasound; ns, nonsignificant.

apoptosis-related proteins in $\mathrm{MiaPaCa}_{2}$ xenograft tumors. Antibodies against activated caspase-3, caspase-8, and Ki-67 were used to examine the effects of HIFU on cell proliferation and apoptosis, respectively, in xenograft tumors (Figure 4). Tumors treated with bufalin or HIFU alone displayed $\sim 30 \%$ or $\sim 40 \%$ of cleaved caspase-3-positive cells $(P<0.001)$, whereas tumors treated with HIFU+bufalin showed $\sim 60 \%$ cleaved caspase-3-positive cells $(P<0.001)$ (Figure 4A and D). Contrasting results were found in caspase-8-stained tumors. Compared with the control cells, bufalin or HIFU treatment alone increased caspase 8 staining cells. What's more, addition of bufalin to HIFU further increased caspase 8 positive cells compared with bufalin or HIFU alone $(P<0.001)$ (Figure $4 \mathrm{~B}$ and $\mathrm{E})$. In contrast with the vehicle control, bufalin or HIFU treatment alone decreased Ki-67 staining to $\sim 12 \%(P<0.05)$ and $\sim 10 \%$ $(P<0.01)$, respectively, whereas HIFU+bufalin treatment reduced Ki67 staining to $\sim 2 \%$ (Figures $4 \mathrm{C}$ and $\mathrm{F}$ ). These data revealed that bufalin enhanced the effect of HIFU by inhibiting cell proliferation and increasing cell apoptosis in $\mathrm{MiaPaCa}_{2}$ xenograft tumors.

\section{Discussion}

Considering the high incidence, rapid progression, and poor prognosis of $\mathrm{PC}$, researchers have recently begun to propose the concept of multidisciplinary comprehensive treatments. A wide variety of disciplines and cooperation treatment have emerged as new avenues for the treatment of advanced PC. For example, intravenous chemotherapy combined with regional arterial infusion chemotherapy can improve treatment efficacy. ${ }^{19,20}$ A minimally invasive treatment, HIFU can be used alone or in combination with chemotherapy to safely and effectively treat patients with advanced PC. ${ }^{21-25}$ Results from a meta-analysis showed that approximately $80 \%$ of patients with advanced PC who received HIFU therapy experienced pain reduction. ${ }^{26} \mathrm{Ji}$ et al ${ }^{27}$ also reported that HIFU treatment was effective and the adverse events (AEs) associated with the treatment were rare and tolerable, especially for old patients and those with poor performance status. ${ }^{28}$ Our previous study was also consistent with these results. ${ }^{10}$

In clinical practice, HIFU treatment is a new non-invasive therapy approved by the China Food and Drug Administration (CFDA) for advanced PC. For elderly patients with poor performance status, or patients unwilling to receive chemotherapy or radiotherapy, HIFU treatment may be considered as the optimal alternative.

As the results of clinical practice have already indicated that HIFU is a safe and effective therapy for the treatment of advanced PC, able to prolong the survival and reduce pain, the mechanism of HIFU has been explored in in vitro and in vivo models. Some researchers insisted that the extrinsic pathway (death receptor-dependent pathway) played a major role in the regulation of apoptosis by HIFU, ${ }^{29}$ whereas some researchers suggested that the mechanism of apoptosis was more dependent on the intrinsic pathway (mitochondriaassociated pathway). ${ }^{30,31}$ Hirokawa et $\mathrm{l}^{32}$ reported that HIFU induced both the extrinsic and intrinsic pathways. In our study, elevated expression of PARP and caspase- 8 was found 
A
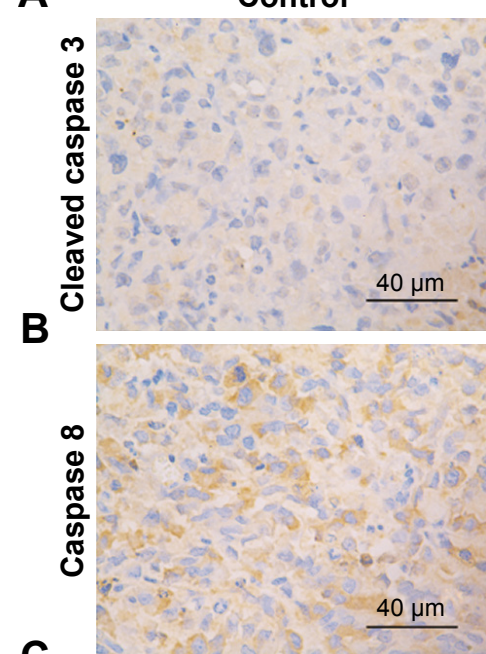

C

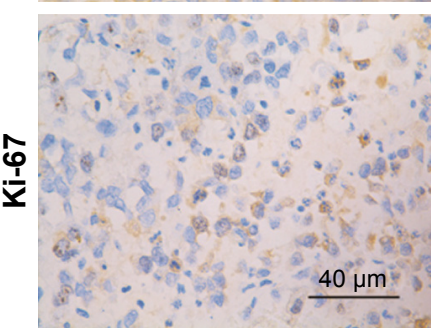

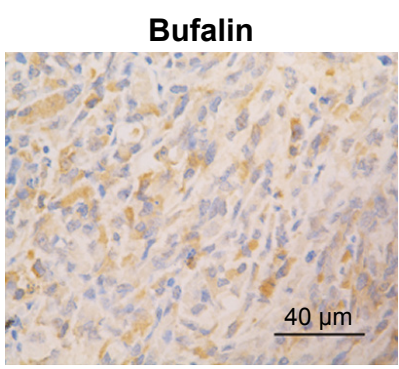
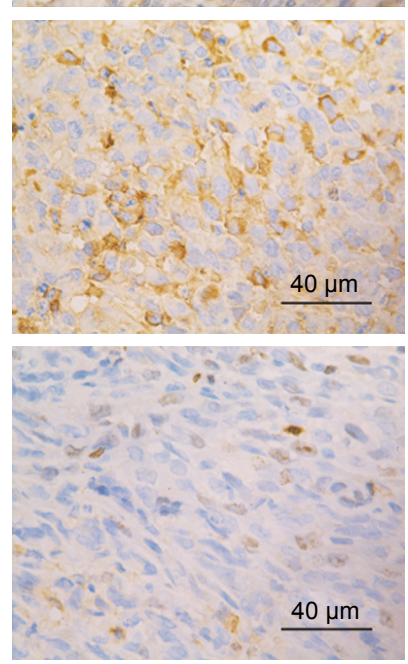

E

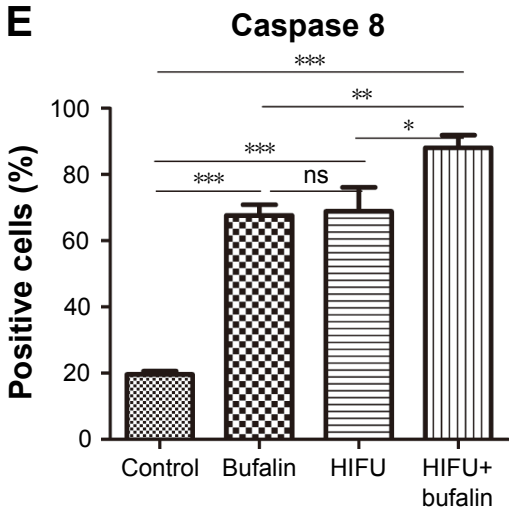

D Cleaved caspase 3

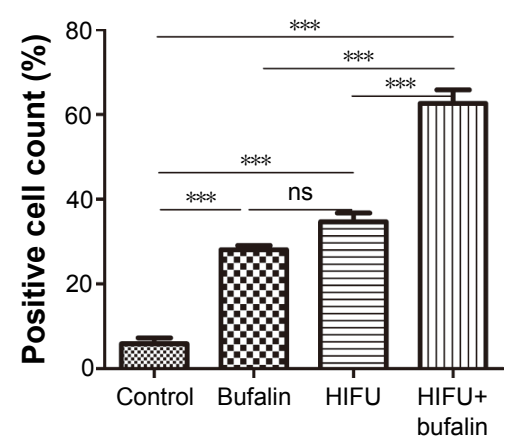

HIFU
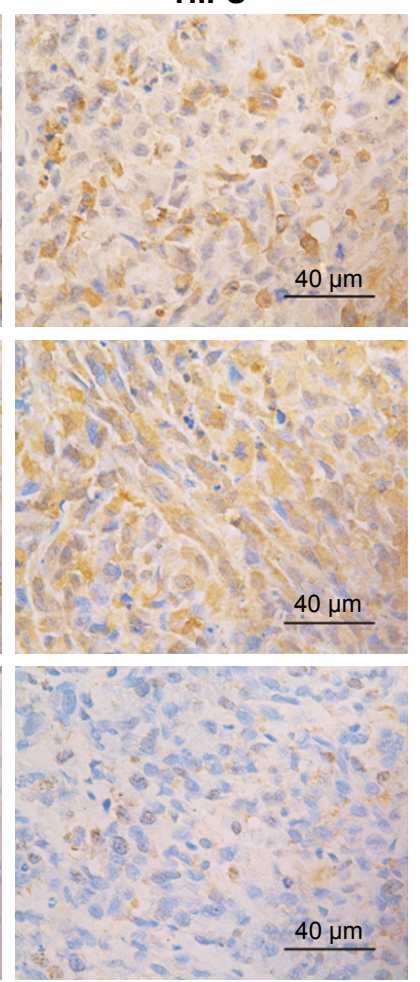

$\mathbf{F}$
HIFU+bufalin

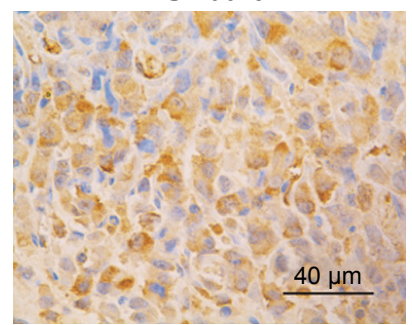

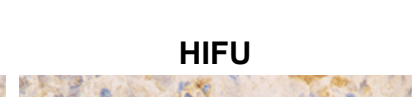
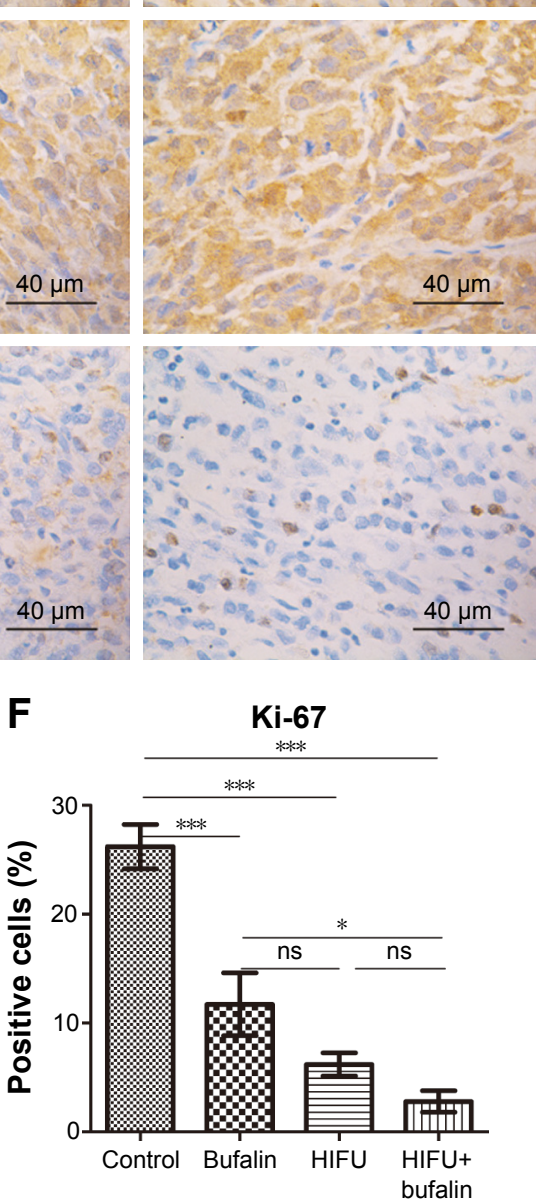

Figure 4 HIFU inhibits cell proliferation and increases cell death in $\mathrm{MiaPaCa}_{2}$ xenograft tumors. Tumors from mice treated with vehicle, bufalin, HIFU, or HIFU+bufalin for 35 days were subjected to immunohistochemical staining with cleaved caspase-3 (A and $\mathbf{D})$, caspase-8 (B and E), and Ki-67 (C and F) antibodies.

Notes: All images are representative of three independent experiments. Scale bar $=50 \mu \mathrm{m}$. $* P<0.05, * * P<0.01$, and $* * * P<0.000$ I, one-way ANOVA.

Abbreviations: HIFU, high-intensity focused ultrasound; ns, nonsignificant.

in the HIFU+bufalin-treated group. These results suggest that apoptosis occurs mainly through the extrinsic pathway.

Based on our results, as PARP was increased after bufalin and HIFU treatment, this may suggest that the apoptotic induction of HIFU and bufalin might not only occur through the extrinsic pathway but also through some other mechanisms. Recently, HIFU treatment with microbubbles was reported. . $^{33,34}$ The temperature of exposed tissue might be higher owing to the enhancement of cavitation by microbubbles, and mechanical destruction by the bubbles might induce cell damage. In addition, bufalin induces death in human cancer cells through the generation of reactive oxygen species, and induces necroptosis through the RIP1/RIP3/PARP-1 pathways. ${ }^{11}$ From these findings, we believe that the microbubbles created by HIFU treatment may increase cell damage, especially to the mitochondria, resulting in the generation of reactive oxygen species. The increased reactive oxygen species induce apoptosis in the cancer cells through different pathways.

\section{Limitations}

There are several limitations to our study. The major limitation was the single drug concentration and HIFU therapeutic 
power; therefore, the optimal combination regime may not have been considered. Second, the mechanism of inducing apoptosis requires further elucidation. Although high expression of caspase- 8 and cleaved caspase- 3 was observed in the treatment groups, the details were unclear. Finally, human pancreatic tumors are more complicated than those generated by animal xenograft models. Therefore, the results require more studies to support clinical trials.

\section{Conclusion}

Our results suggest that it is important to adjust the drug concentration and optimize the therapeutic power of HIFU when applying combination treatments in future studies.

\section{Acknowledgment}

This study was funded by the National Natural Science Foundation of China (grant numbers 81573753 and 81774063).

\section{Disclosure}

The authors report no conflicts of interest in this work.

\section{References}

1. Siegel RL, Miller KD, Jemal A, Statistics C. Cancer statistics, 2017. CA Cancer J Clin. 2017;67(1):7-30.

2. Rahib L, Smith BD, Aizenberg R, Rosenzweig AB, Fleshman JM, Matrisian LM. Projecting cancer incidence and deaths to 2030: the unexpected burden of thyroid, liver, and pancreas cancers in the United States. Cancer Res. 2014;74(11):2913-2921.

3. Shaib WL, Jones JS, Goodman M, et al. Evaluation of treatment patterns and survival outcomes in elderly pancreatic cancer patients: a surveillance, epidemiology, and end results-medicare analysis. Oncologist. 2018;23(6):704-711

4. Regine WF, Winter KA, Abrams R, et al. Fluorouracil-based chemoradiation with either gemcitabine or fluorouracil chemotherapy after resection of pancreatic adenocarcinoma: 5-year analysis of the U.S. Intergroup/RTOG 9704 phase III trial. Ann Surg Oncol. 2011;18(5): 1319-1326.

5. Dong X, Yang Z. High-intensity focused ultrasound ablation of uterine localized adenomyosis. Curr Opin Obstet Gynecol. 2010;22(4):326-330.

6. Yang Z, Cao YD, Hu LN, Wang ZB. Feasibility of laparoscopic highintensity focused ultrasound treatment for patients with uterine localized adenomyosis. Fertil Steril. 2009;91(6):2338-2343.

7. Wang W, Wang Y, Tang J. Safety and efficacy of high intensity focused ultrasound ablation therapy for adenomyosis. Acad Radiol. 2009;16(11): 1416-1423.

8. Kim SH, Jung SE, Kim HL, Hahn ST, Park GS, Park WC. The potential role of dynamic MRI in assessing the effectiveness of high-intensity focused ultrasound ablation of breast cancer. Int J Hyperthermia. 2010; 26(6):594-603.

9. Wu F, Wang ZB, Zhu H, et al. Extracorporeal high intensity focused ultrasound treatment for patients with breast cancer. Breast Cancer Res Treat. 2005;92(1):51-60.

10. Ning ZY, Cheng CS, Xie J, et al. A retrospective analysis of survival factors of high intensity focused ultrasound (HIFU) treatment for unresectable pancreatic cancer. Discov Med. 2016;21(118):435-445.

11. Li Y, Tian X, Liu X, Gong P. Bufalin inhibits human breast cancer tumorigenesis by inducing cell death through the ROS-mediated RIP1/ RIP3/PARP-1 pathways. Carcinogenesis. 2018;39(5):700-707.
12. Sheng X, Zhu P, Qin J, Li Q. The biological role of autophagy in regulating and controlling the proliferation of liver cancer cells induced by bufalin. Oncol Rep. 2018;39(6):2931-2941.

13. Sun J, Xu K, Qiu Y, et al. Bufalin reverses acquired drug resistance by inhibiting stemness in colorectal cancer cells. Oncol Rep. 2017;38(3): $1420-1430$.

14. Xiang RF, Wang Y, Zhang N, et al. MK2206 enhances the cytocidal effects of bufalin in multiple myeloma by inhibiting the AKT/mTOR pathway. Cell Death Dis. 2017;8(5):e2776.

15. Zhakeer Z, Hadeer M, Tuerxun Z, Tuerxun K. Bufalin inhibits the inflammatory effects in asthmatic mice through the suppression of nuclear factor-kappa B activity. Pharmacology. 2017;99(3-4):179-187.

16. Zhao H, Li Q, Pang J, Jin H, Li H, Yang X. Blocking autophagy enhances the pro-apoptotic effect of bufalin on human gastric cancer cells through endoplasmic reticulum stress. Biol Open. 2017;6(10):1416-1422.

17. Wang H, Zhang C, Xu L, et al. Bufalin suppresses hepatocellular carcinoma invasion and metastasis by targeting HIF-1 $\alpha$ via the PI3K/ Akt/mTOR pathway. Oncotarget. 2016;7(15):20193-20208.

18. Yi J, Wu L, Liu Z, et al. High-intensity focused ultrasound ablation induced apoptosis in human hepatocellular carcinoma. Hepatogastroenterology. 2014;61(136):2336-2339.

19. Homma H, Doi T, Mezawa S, et al. A novel arterial infusion chemotherapy for the treatment of patients with advanced pancreatic carcinoma after vascular supply distribution via superselective embolization. Cancer. 2000;89(2):303-313.

20. Azizi A, Naguib NN, Mbalisike E, Farshid P, Emami AH, Vogl TJ. Liver metastases of pancreatic cancer: role of repetitive transarterial chemoembolization (TACE) on tumor response and survival. Pancreas. 2011;40(8):1271-1275.

21. Li PZ, Zhu SH, He W, et al. High-intensity focused ultrasound treatment for patients with unresectable pancreatic cancer. Hepatobiliary Pancreat Dis Int. 2012;11(6):655-660.

22. Lee JY, Choi BI, Ryu JK, et al. Concurrent chemotherapy and pulsed high-intensity focused ultrasound therapy for the treatment of unresectable pancreatic cancer: initial experiences. Korean J Radiol. 2011;12(2): $176-186$.

23. Marinova M, Huxold HC, Henseler J, et al. Clinical effectiveness and potential survival benefit of US-Guided high-intensity focused ultrasound therapy in patients with advanced-stage pancreatic cancer. Ultraschall Med. Epub 2018 Apr 17.

24. Zhao J, Zhao F, Shi Y, Deng Y, Hu X, Shen H. The efficacy of a new high intensity focused ultrasound therapy for locally advanced pancreatic cancer. J Cancer Res Clin Oncol. 2017;143(10):2105-2111.

25. Rischmann P, Gelet A, Riche B, et al. Focal high intensity focused ultrasound of unilateral localized prostate cancer: a prospective multicentric Hemiablation study of 111 patients. Eur Urol. 2017;71(2):267-273.

26. Dababou S, Marrocchio C, Rosenberg J, et al. A meta-analysis of palliative treatment of pancreatic cancer with high intensity focused ultrasound. J Ther Ultrasound. 2017;5(1):9.

27. Ji Y, Zhang Y, Zhu J, et al. Response of patients with locally advanced pancreatic adenocarcinoma to high-intensity focused ultrasound treatment: a single-center, prospective, case series in China. Cancer Manag Res. 2018;10:4439-4446.

28. Marinova M, Strunk HM, Rauch M, et al. High-intensity focused ultrasound (HIFU) for tumor pain relief in inoperable pancreatic cancer: evaluation with the pain sensation scale (SES)]. Schmerz. 2017;31(1):31-39.

29. Tang W, Liu Q, Wang X, et al. Involvement of caspase 8 in apoptosis induced by ultrasound-activated hematoporphyrin in sarcoma 180 cells in vitro. J Ultrasound Med. 2008;27(4):645-656.

30. Luo W, Zhou X, Gong X, Zheng M, Zhang J, Guo X. Study of sequential histopathologic changes, apoptosis, and cell proliferation in rabbit livers after high-intensity focused ultrasound ablation. J Ultrasound Med. 2007;26(4):477-485.

31. Firestein F, Rozenszajn LA, Shemesh-Darvish L, Elimelech R, Radnay J, Rosenschein U. Induction of apoptosis by ultrasound application in human malignant lymphoid cells: role of mitochondria-caspase pathway activation. Ann N Y Acad Sci. 2003;1010:163-166. 
32. Hirokawa N, Koito K, Okada F, et al. High-intensity focused ultrasound induced apoptosis with caspase 3,8 , and 9/6 activation in rat hepatoma. J Med Ultrason. 2009;36(4):177-185.

33. Yang D, Ni Z, Yang Y, et al. The enhanced HIFU-induced thermal effect via magnetic ultrasound contrast agent microbubbles. Ultrason Sonochem. 2018;49:111-117.
34. Yu MH, Lee JY, Kim HR, et al. Therapeutic effects of microbubbles added to combined high-intensity focused ultrasound and chemotherapy in a pancreatic cancer xenograft model. Korean J Radiol. 2016;17(5): 779-788.

\section{Publish your work in this journal}

OncoTargets and Therapy is an international, peer-reviewed, open access journal focusing on the pathological basis of all cancers, potential targets for therapy and treatment protocols employed to improve the management of cancer patients. The journal also focuses on the impact of management programs and new therapeutic agents and protocols on

\section{Dovepress}

patient perspectives such as quality of life, adherence and satisfaction. The manuscript management system is completely online and includes a very quick and fair peer-review system, which is all easy to use. Visit http://www.dovepress.com/testimonials.php to read real quotes from published authors.

Submit your manuscript here: http://www.dovepress.com/oncotargets-and-therapy-journal 